\title{
DYNAMICS OF THE OPTICAL CHARACTERISTICS OF EROSION LASER FLARES OF METALS FORMED BY INTENSE NANOSECOND LASER PULSES UNDER ATMOSPHERIC CONDITIONS
}

\author{
V. K. Goncharov, K. V. Kozadaev*, and D. V. Shchegrikovich \\ UDC 621.373.826.004.14 \\ Time resolved laser induced plasma spectroscopy is used to study the erosion laser flares of a number of metals (Ni, \\ $\mathrm{Pb}, \mathrm{Zn})$ exposed to intense 20- $\mathrm{ns} \mathrm{Nd}: \mathrm{YAG}$ laser pulses in air at atmospheric pressure $(\lambda=1064 \mathrm{~nm}, 0.1 \leq q \leq 3 \mathrm{GW} /$ \\ $\mathrm{cm}^{2}$ ). The time variation of the emission from the laser induced plasma is studied. Characteristic lines of the ele- \\ ments are found in the laser flares and the formation of the spectral structure of the erosion laser flares under these \\ conditions is discussed.
}

Keywords: laser induced plasma spectroscopy of metals, erosion laser flare, integrated plasma luminosity, electron temperature, electron density.

Introduction. Laser technologies are used effectively in many areas: metal processing, communications, medicine, military technology, etc. Laser processing of materials is currently a highly developed branch of industry and is constantly being improved [1-5]. The ability to obtain a high concentration of optical radiation within narrow spatial, temporal, and spectral ranges has opened up new possibilities for local surface and bulk processing of materials, contactless diagnostics and control of fast processes, precision measurements, etc.

Operational control of the energy, spectral, spatial, and temporal characteristics of transient plasmas produced by laser irradiation is of great practical interest for the development of new precision technologies for laser processing of metals using modern repetitively pulsed lasers. Laser induced breakdown/plasma spectroscopy (LIBS/LIPS) is an analytic technique that is currently under rapid development and can be used for spectroscopic studies of plasmas with high spectral and spatial resolution [6]. The use of time resolved laser spectroscopy (TRLS), along with LIPS techniques, has made it possible to study the temporal structure of the plasma radiation [7,8]. This, in turn, makes it possible to study the complete physical picture of the processes taking place in erosion laser flares (ELF) of metals in more detail. Studies of the dynamics of the integrated emission from the ELF may also be of considerable practical interest $[9,10]$.

This is a study of the dynamics (time variation) and structure of the optical characteristics of ELF produced during metal processing by intense 20-ns optical pulses. This is done using a software and hardware system with high spectral and temporal resolution.

Experiment. The experimental apparatus developed for studying the dynamics and structure of the optical characteristics of metal ELF under normal atmospheric conditions is illustrated in Fig. 1. In terms of function this system can be divided into several units: for generating the driver pulses, recording the optical characteristics of the ELF, and synchronization. The driver pulse generator includes a commercial Nd:YAG repetitively pulsed laser (Lotis 2137) and sensors for measuring the source pulse characteristics (bolometer 3 (IMO-3) and photodiode 5) manufactured in Belarus. The laser pulse duration is $20 \mathrm{~ns}$, its wavelength is $1064 \mathrm{~nm}$, and the energy per pulse ranges up to $500 \mathrm{~mJ}$, which when focussed onto a 1-mm-diam spot yields an irradiance of $\sim 3 \mathrm{GW} / \mathrm{cm}^{2}$.

The unit for recording the optical characteristics of the ELF includes an MS2001i monochromator-spectrograph with a CCD strip as the optical sensor, an MDR-12 monochromator together with a silicon photomultiplier (SPM 10020 SensL), and a photodiode 6 (FD-21-KP) mounted in a vertical plane orthogonal to the target plane. The measured parameters

\footnotetext{
${ }^{*}$ To whom correspondence should be addressed.
}

A. N. Sevchenko Institute of Applied Physics Problems, Belarusian State University, 7 Kurchatov Str., Minsk, 220108, Belarus; e-mail: kozadaeff@mail.ru. Original article submitted September 27, 2012. Translated from Zhurnal Prikladnoi Spektroskopii, Vol. 80, No. 3, pp. 409-416, May-June, 2013. 


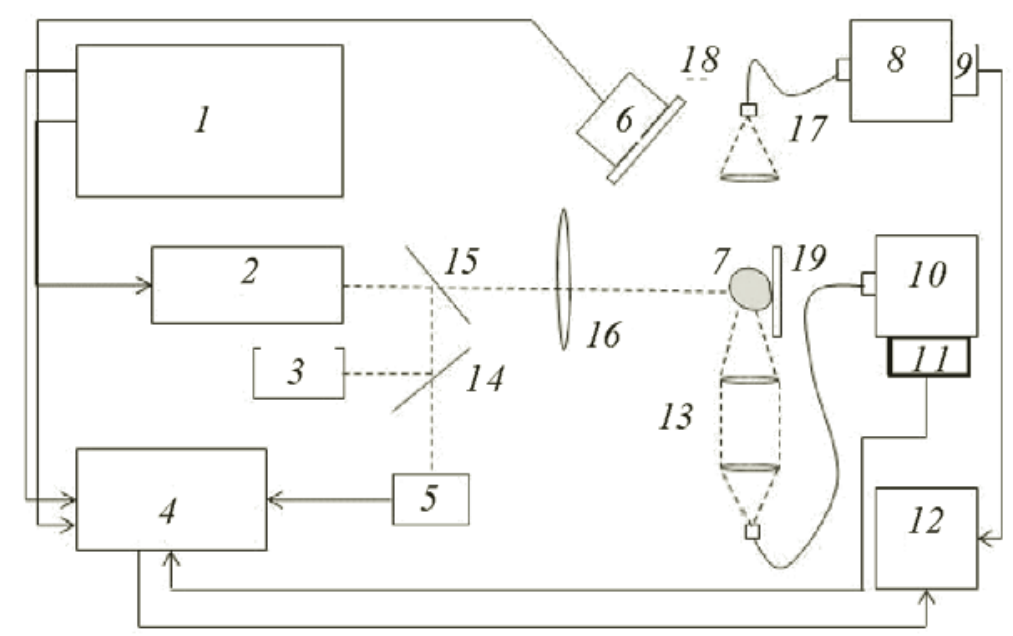

Fig. 1. Conceptual diagram of the experimental apparatus: (1) pulse delay generator, (2) laser, (3) calorimeter, (4) ADC, (5, 6) photodiodes, (7) erosion laser flare, (8) spectrograph, (9) CCD strip, (10) monochromator, (11) photomultiplier, (12) personal computer, (13) optical condenser, $(14,15)$ steering plates, $(16,17)$ focussing lenses, $(18)$ interference filter $(\lambda=1064 \mathrm{~nm})$, (19) metal target.

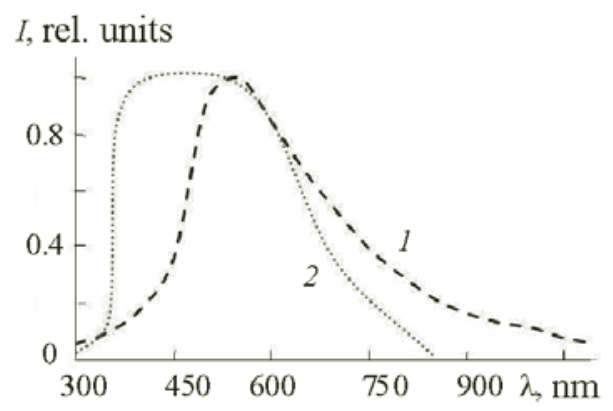

Fig. 2. Spectral sensitivity of the photodetectors in the system: photomultiplier (1) and photodiode (2).

are digitized with a high-speed 4-channel ADC (B422 digital oscilloscope) and processes using a personal computer with specialized programs. The sampling rate of the $\mathrm{ADC}$ is $100 \mathrm{MHz}$

To ensure regular operation of all the components of the parts of this apparatus, a G200P multichannel pulse delay generator was used. It generates synchronized pulses with a controllable time delay for each of the units. The relative spectral sensitivities of the photodetectors are shown in Fig. 2 [11].

With this apparatus it was possible to study the following optical characteristics of the ELF: (a) the time variation of the spectrally integrated emission of the ELF (with the spectral sensitivity of the photodetector (Fig. 2) taken into account) with high temporal resolution (10 ns); (b) the time integrated spectral structure of the ELF with high spectral resolution (0.12 $\mathrm{nm}$ ); and, (c) the time variation of the intensity of individual lines in the spectrum of the ELF with high spectral and temporal resolution $(0.5 \mathrm{~nm}$ and $10 \mathrm{~ns})$.

Lead, zinc, and nickel were chosen as metal targets because the substantial differences in their optical and thermal characteristics (see Table 1) make it possible to speak of laser erosion processes for metals as a whole. This is because zinc has the lowest difference between its melting and boiling points with a relatively low heat of vaporization, while lead is the most easily melted but has a high boiling point, and nickel has high melting and boiling temperatures and a high specific heat 
TABLE 1. Thermal Characteristics* of the Metals [12]

\begin{tabular}{|c|c|c|c|c|c|}
\hline Metal & $T_{\mathrm{m}},{ }^{\circ} \mathrm{C}$ & $Q_{\mathrm{m}}, \mathrm{kJ} /$ mole & $T_{\text {vap }}{ }^{\circ} \mathrm{C}$ & $Q_{\text {vap }}, \mathrm{kJ} / \mathrm{mole}$ & $\alpha(\lambda=1000 \mathrm{~nm}), \%$ \\
\hline $\mathrm{Ni}$ & 1455 & 17.8 & 2800 & 380.6 & 72 \\
$\mathrm{~Pb}$ & 327 & 4.772 & 1751 & 179.5 & 80 \\
$\mathrm{Zn}$ & 420 & 7.28 & 907 & 114.7 & 69 \\
\hline
\end{tabular}

${ }^{*} T_{\mathrm{m}}$ and $T_{\mathrm{vap}}$ are the melting and boiling temperatures, $Q_{\mathrm{m}}$ and $Q_{\mathrm{vap}}$ are the specific heats of melting and vaporization, and $\alpha(\lambda=1000 \mathrm{~nm})$ is the reflectivity at a wavelength of $1000 \mathrm{~nm}$.

of vaporization. These metals have similar reflectivities, i.e., initially they absorb roughly the same fraction of energy from the driver pulse $[9,10]$.

Results and Discussion. The formation of metal ELF during irradiation by 20-ns, high-irradiance laser pulses $\left(10^{8}-10^{10} \mathrm{~W} / \mathrm{cm}^{2}\right)$ differs qualitatively from the cases of millisecond (and longer) pulses with a moderate irradiance $\left(10^{5}-10^{8}\right.$ $\left.\mathrm{W} / \mathrm{cm}^{2}\right)[1-5,13]$ and high irradiance femto- and picosecond pulses [14-16]. However, there are some similarities in the laser erosion processes and the evolution of the plasma compared to high irradiance pulses with durations of $\sim 100 \mathrm{~ns}[9,10,17,18]$.

Based on the features of laser erosion of metals by submicrosecond (100 ns) high intensity pulses, we may conclude that $\sim 50 \mathrm{~ns}$ after the onset of the laser pulse (a time comparable to the risetime of the driver pulse) an intensely luminous plasma develops in the surface region of the target. A laser flare propagates perpendicular to the target surface, absorbs almost all the energy in the trailing edge of the laser pulse by inverse bremsstrahlung and, thereby, is heated substantially. The characteristic propagation speed of the plasma at the onset of the pulse is $7-20 \mathrm{~km} / \mathrm{s}$, depending on the type of metal target and the beam irradiance.

After the intensity of the driver pulse falls off a fairly slow cooling of the flare takes place, primarily through adiabatic expansion of the plasma, as indicated by a slight delay in the luminosity of the flare relative to the trailing edge of the driver pulse. Here a scattered component shows up in the extinction structure of probe light that is indicative of the formation of local plasma inhomogeneities in the flare which determine the passage of the probe light through the flare at this time. Subsequently, 2.0-2.5 $\mu \mathrm{s}$ after the start of the laser pulse absorption of the probe radiation again begins to predominate over scattering with a fairly low level of loss in the flare, which indicates the onset of finely dispersed droplet formation owing to condensation of the plasma flare. This process continues for a fairly long time $(\sim 300-400 \mu \mathrm{s})$ after the laser pulse and leads to the appearance of nanosized metal particles in the surface region of the metal.

Data from a study of the time distribution of the integrated light from ELF produced by 20-ns laser pulses with different irradiances on metal targets are shown in Fig. 3. The following behavior occurs for all these metals. The maximum luminosity of the ELF at all irradiances is delayed relative to the peak of the laser intensity by 20-60 ns. This shows that the entire leading edge of the driver laser pulse reaches the target surface unimpeded.

When the irradiance of the laser pulse is increased to $1 \mathrm{GW} / \mathrm{cm}^{2}$ a significant increase in the overall luminosity is observed, together with a significant persistence of the trailing edge of the pulse of ELF luminosity; this is indicative of a substantial increase in the maximum plasma density above the levels for lower laser intensities [19]. The shape of the ELF luminosity pulse is similar for all the materials studied here; this suggests that the laser erosion processes are qualitatively the same for these metals. All of these curves have similar leading edges related to the rate of arrival of the radiant energy (the steepness of the leading edge of the driver pulse). The intensity of the plasma luminosity is highest for lead; this may be explained by its lower melting point (see Table 1). The time variation of the intensity drop in the trailing edge of the luminous pulse has a distinctively individual character for each of the metals. Thus, the plasma flare of the $\mathrm{Zn}$ target relaxes most rapidly. The trailing edges of the ELF luminosity of $\mathrm{Ni}$ and $\mathrm{Pb}$ are stretched out significantly relative to the driver pulse, which indicates that the adiabatic cooling of the ELF is fairly slow [19].

The optical spectra of the ELF from these metals for different irradiances are shown in Fig. 4. Also shown for comparison is a typical breakdown spectrum for air by a laser pulse with the same characteristics. It should be noted that the spectra of Fig. 4 are normalized, since these curves have different intensity levels; for example, the maximum intensity of the optical breakdown spectrum for air is two orders of magnitude lower than that of the ELF for lead.

The metal ELF spectra typically consist of lines, sometimes accompanied by a continuum component at $\sim 400 \mathrm{~nm}$ mainly from the luminosity of the air plasma (Fig. 4d). The contribution of the air plasma to the $\mathrm{Zn}$ and Pb ELF spectra is 


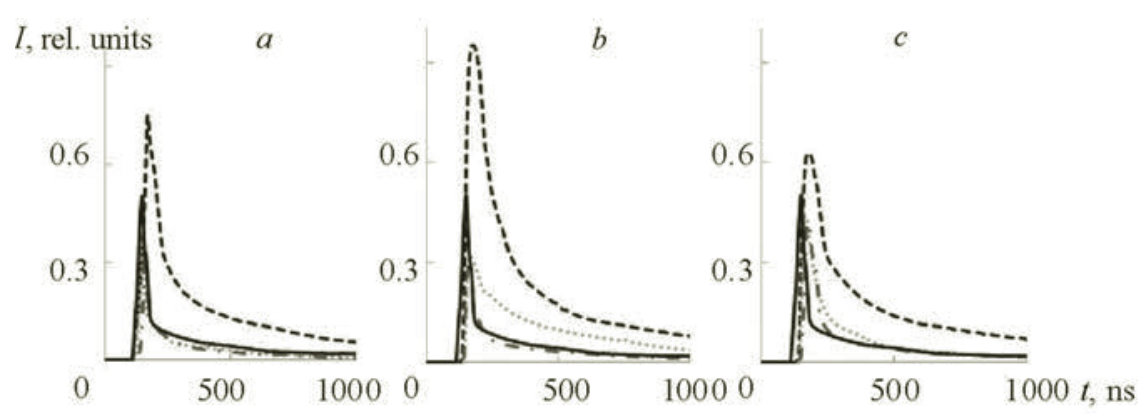

Fig. 3. Time variation of the integrated luminosity of erosion laser flares for $\mathrm{Zn}(\mathrm{a}), \mathrm{Pb}$ (b), and Ni (c); $I=10^{9}$ (dashed curve), $3 \cdot 10^{8}$ (dotted curve), $10^{8} \mathrm{~W} / \mathrm{cm}^{2}$ (dot-dashed curve); the smooth curve is the driver laser pulse.

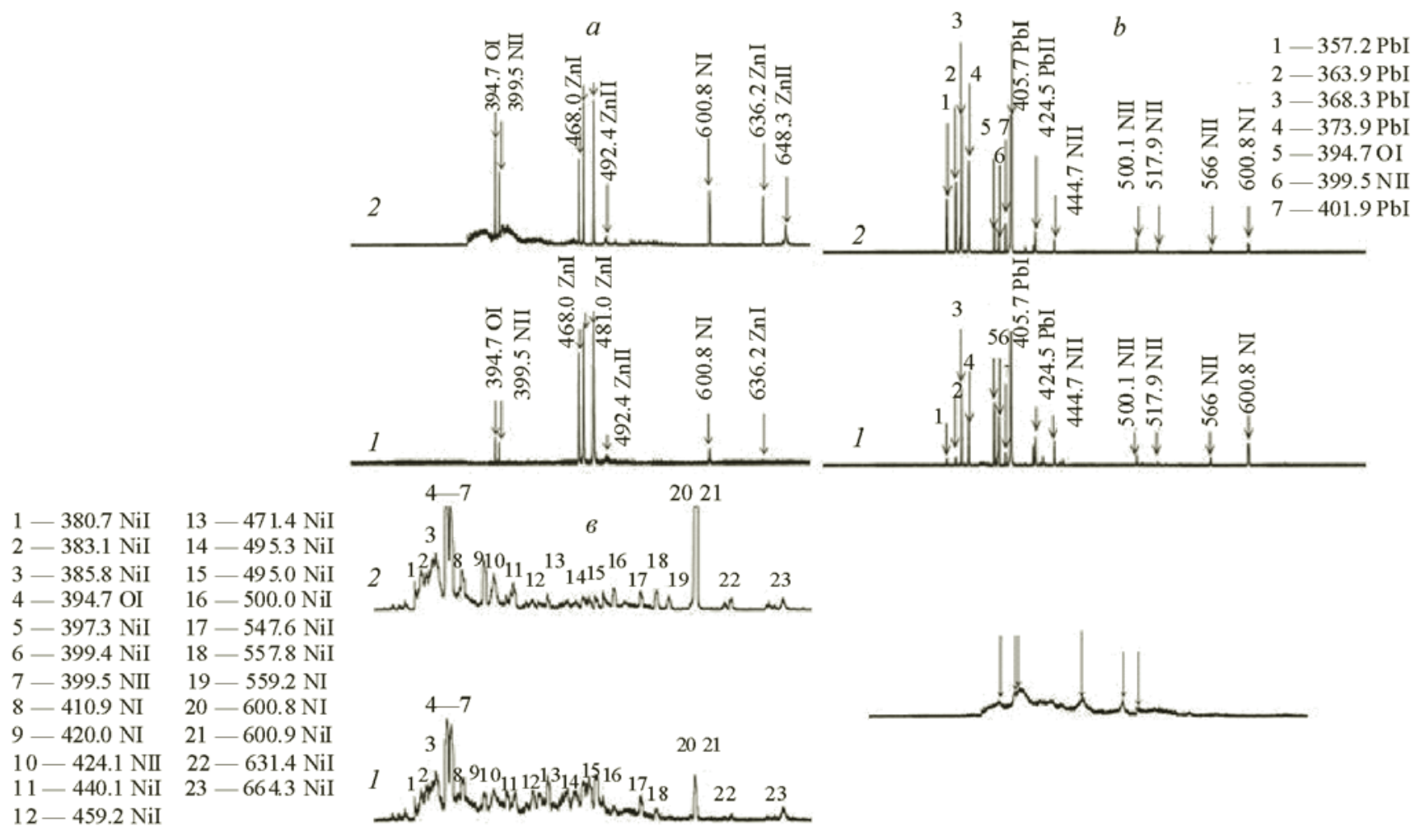

Fig. 4. Optical spectra of ELF for $\mathrm{Zn}(\mathrm{a}), \mathrm{Pb}(\mathrm{b})$, and $\mathrm{Ni}$ (c) for irradiances of $10^{8}$ (1) and $10^{9} \mathrm{~W} / \mathrm{cm}^{2}(2)$; the spectrum of an optical breakdown in air (d).

negligible. In the Ni spectrum, intense lines around $\sim 400 \mathrm{~nm}$ are superimposed on the air breakdown plasma, so the estimated contribution of the metal lines and atmospheric lines is less evident in this case. All the metal ELF spectra are accompanied by the fairly bright N I $600.8 \mathrm{~nm}$ line which hardly shows up in the emission from the air breakdown plasma. It seems that this line appears in the metal ELF spectra as part of the formation and evolution of the metal plasma flares under these laser interaction conditions. (The N I $600.8 \mathrm{~nm}$ line and the bright Ni I $600.9 \mathrm{~nm}$ line occupy the same position in the spectrum of the Ni ELF.) 
TABLE 2. Calculated Electron Temperatures and Densities and Some Characteristics of the Spectral Lines

\begin{tabular}{|c|c|c|c|c|c|c|c|c|}
\hline \multirow[b]{2}{*}{ Line } & \multirow[b]{2}{*}{ Electron configuration } & \multirow[b]{2}{*}{$g$} & \multirow[b]{2}{*}{$f$} & \multirow[b]{2}{*}{ E } & \multicolumn{2}{|c|}{$I=10^{8} \mathrm{~W} / \mathrm{cm}^{2}$} & \multicolumn{2}{|c|}{$I=10^{9} \mathrm{~W} / \mathrm{cm}^{2}$} \\
\hline & & & & & $\begin{array}{l}T_{e} \\
\mathrm{eV}\end{array}$ & $n_{e}, \mathrm{~cm}^{-3}$ & $T_{e}, \mathrm{eV}$ & $n_{e}, \mathrm{~cm}^{-3}$ \\
\hline $\mathrm{PbI} 368.3 \mathrm{~m}$ & $6 p_{1 / 2} 7 s_{1 / 2}(1 / 2.1 / 2)^{0} \rightarrow 6 p_{1 / 2} 6 p_{3 / 2}(1 / 2.3 / 2)$ & 3 & 0.64 & 4.34 & \multirow{2}{*}{0.50} & \multirow{2}{*}{$1.2 \cdot 10^{14}$} & \multirow{2}{*}{0.55} & \multirow{2}{*}{$3.7 \cdot 10^{14}$} \\
\hline $\mathrm{PbI} 374.0 \mathrm{~nm}$ & $6 p_{3 / 2} 7 s_{1 / 2}(3 / 2.1 / 2)^{\mathrm{o}} \rightarrow 6 p_{3 / 2}(3 / 2.3 / 2)$ & 5 & 5.3 & 5.97 & & & & \\
\hline $\mathrm{ZnI} 481.0 \mathrm{~nm}$ & $4 s 5 s^{3} S \rightarrow 4 s 4 p^{3} P^{0}$ & 3 & 7.2 & 6.66 & \multirow{2}{*}{0.33} & \multirow{2}{*}{$7.3 \cdot 10^{13}$} & \multirow{2}{*}{0.58} & \multirow{2}{*}{$4.5 \cdot 10^{14}$} \\
\hline $\mathrm{ZnI} 636.2 \mathrm{~nm}$ & $4 s 4 d^{1} D \rightarrow 4 s 4 p{ }^{1} P^{\mathrm{o}}$ & 5 & 2.8 & 7.74 & & & & \\
\hline NiI $440.1 \mathrm{~nm}$ & & 5 & 6.8 & 6.00 & \multirow{2}{*}{0.31} & \multirow{2}{*}{$1.1 \cdot 10^{13}$} & \multirow{2}{*}{0.42} & \multirow{2}{*}{$2.5 \cdot 10^{13}$} \\
\hline $\mathrm{NiI} 459.2 \mathrm{~nm}$ & & 5 & 2.0 & 6.29 & & & & \\
\hline
\end{tabular}

The spectral structure of the emission from laser induced plasmas under similar conditions have been studied previously [20-24]. Spectral diagnostics of a Pb laser erosion plasma in vacuum [20] with laser pulse parameters of $I=5 \cdot 10^{8}$ $\mathrm{W} / \mathrm{cm}^{2}, \lambda=1064 \mathrm{~nm}$, and $\tau=20$ yielded and estimated electron temperature $T_{e}$ of 0.86 and $0.48 \mathrm{eV}$ at distances of 1 and 7 $\mathrm{mm}$ from the target, respectively, based on a comparison of the intensities of the $\mathrm{Pb}$ I 364, 368.3, and $405.7 \mathrm{~nm}$ lines. A Ge laser plasma in vacuum has been studied [21] under similar irradiation conditions [20]. There the electron temperature was estimated to be $T_{e}=0.6-0.7 \mathrm{eV}$. The processes in laser erosion Cu plasmas have been examined [22] for pulse parameters $I=$ $4.5 \cdot 10^{8} \mathrm{~W} / \mathrm{cm}^{2}, \lambda=355 \mathrm{~nm}$, and $\tau=6 \mathrm{~ns}$ under atmospheric conditions. It was found that with the passage of time (from 200 ns to $2 \mu \mathrm{s}$ after the onset of the interaction) the estimated electron temperature $T_{e}$ varied from 0.84 to $0.69 \mathrm{eV}$ with a decrease in the electron density from $1.1 \cdot 10^{15}$ to $4.5 \cdot 10^{13} \mathrm{~cm}^{-3}$. Laser $\mathrm{W}$ plasmas in vacuum have been studied [23] with laser pulse parameters $I \leq 30 \cdot 10^{9} \mathrm{~W} / \mathrm{cm}^{2}, \lambda=1064 \mathrm{~nm}$, and $\tau=3 \mathrm{~ns}$. In this case the estimated electron temperature of the plasma was as high as $1.2 \mathrm{eV}$ and the electron density, up to $10^{17} \mathrm{~cm}^{-3}$. In a study [24] of laser induced $\mathrm{Ag}, \mathrm{Au}$, and $\mathrm{Cu}$ plasmas in water with double pulses $\left(I=(0.1-5.0) \cdot 10^{9} \mathrm{~W} / \mathrm{cm}^{2}, \lambda=532 \mathrm{~nm}\right.$, and $\left.\tau=10 \mathrm{~ns}\right)$ the electron temperature and density were estimated to be $0.8-1.4 \mathrm{eV}$ and $(1-2) \cdot 10^{18} \mathrm{~cm}^{-3}$, respectively.

For determining the electron temperature by comparing relative line strengths it is sufficient to have partial local thermodynamic equilibrium (LTE) of the populations of the corresponding particles. It has been shown [25] that this condition can be satisfied for a homogeneous transient hydrogen plasma with a fairly low electron density $n_{e} \sim 10^{14} \mathrm{~cm}^{-3}$. In the papers cited above [20-24] with similar experimental parameters, the condition for partial LTE was satisfied, so that $T_{e}$ and $n_{e}$ were estimated in this paper using the approximation of partial LTE $[25,26]$. In this case, we have [25]

$$
k T_{e}=\left(E_{2}-E_{1}\right) / \ln \left(\frac{Q_{1} \lambda_{1}^{3} g_{2} f_{2}}{Q_{2} \lambda_{2}^{3} g_{1} f_{1}}\right),
$$

where $E_{i}, Q_{i}, \lambda_{i}, g_{i}$, and $f_{i}$ are, respectively, the excitation energy, total intensity, wavelength, statistical weight of the lower level, and oscillator strength for the $i$-th spectrum line of the element.

The electron density can be estimated using the formula [21]

$$
n_{e}=\left(8.75 \cdot 10^{-27} z^{3} t_{\mathrm{r}} e^{-9 / 2}\right)^{-1 / 2},
$$

where $z$ and $t_{\mathrm{r}}$ are the charge and recombination time of the ion.

The electron temperatures and densities calculated using the observed spectra (Fig. 4) and some parameters of the spectrum lines [27-30] are listed in Table 2. These ELF spectra were taken at a height of $3 \mathrm{~mm}$ from the target surface, i.e., in the region where the plasma expands adiabatically. Table 2 shows that the estimated electron temperatures and densities of the ELF for these metals are basically consistent with the values obtained in the earlier work [20-24]. The obvious tendency for these characteristics to increase with rising laser intensity is retained. In addition, we can say that the ELF of the more refractory $\mathrm{Ni}$ (see Table 1) has lower electron temperature and density than those of $\mathrm{Pb}$ and $\mathrm{Zn}$. 

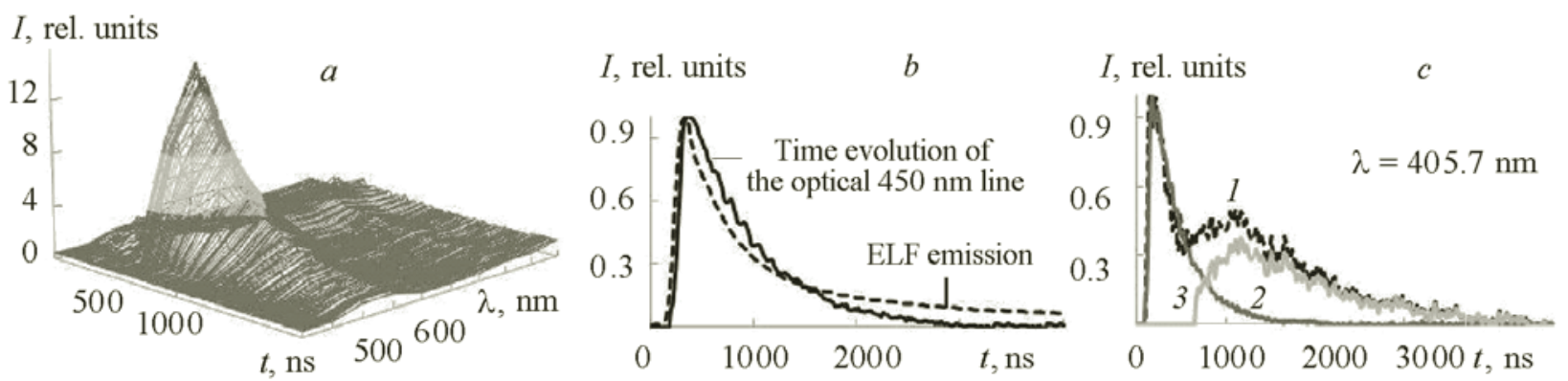

Fig. 5. Time evolution of individual spectrum lines of the ELF.

The next step is to study the temporal structure of the individual spectrum lines of the ELF. A monochromator equipped with a photomultiplier and a high-speed ADC was used to visualize the time evolution of the ELF luminosity with high time resolution within narrow spectral bands $(\Delta \lambda \sim 0.5 \mathrm{~nm})$; these observations were made at the wavelengths of strong metal lines as well as in regions of the spectra with no features. The behavior was qualitatively similar for all of the metals. Figure 5 shows some data for just one irradiation condition $\left(I=1 \mathrm{GW} / \mathrm{cm}^{2}\right)$ with a Pb target. There are two types of time dependence for the emission within individual spectral intervals for these metals. The first is ELF emission in regions of the spectrum with no characteristic narrow lines of the metal or the air (Fig. 5b). It is clear that the time dependence of the emission for this segment of the spectrum follows the time dependence of the integrated emission from the lead ELF (Fig. 3b, dashed curve). The second type includes ELF emission in spectral intervals corresponding to bright lines of the metals or the air, as shown in Fig. 4b. The time evolution of the emission from these lines is illustrated in Fig. 5c. This curve is a superposition of two processes: an initial emission with the same structure as the integrated emission (Fig. 5c, curve 2) and a longer lasting emission in the characteristic line with a delay of 600-700 ns relative to the laser pulse owing to recombination of the corresponding ions (Fig. 5c, curve 3). The sum of these contributions gives the profile of the emission at this line over time (Fig. 5c, curve 1).

In order to reveal the spectral characteristics of the first type of time dependence the entire visible range of the ELF spectrum (300-800 nm) was scanned over equal intervals. In order to compensate for the spectral sensitivity of the photomultiplier (Fig. 2), the curves for each segment of the spectrum were scaled. A three dimensional graph showing the combined spectral and time variations of the intensity a lead ELF plasma is shown in Fig. 5a. The emission peak of the metal ELF coincides with the maximum of the integrated emission (Fig. 3b) and is localized within a rather wide spectral region $(400-500 \mathrm{~nm})$.

The analogous processes in rarefied gas atmospheres ( $100 \mathrm{~Pa})$ for Ti, $\mathrm{Al}$, and $\mathrm{Fe}$ targets are discussed in [31]. This book dealt with a somewhat different problem - the justification of laser erosion of materials as an effective source of data for spectral analysis. Nevertheless, some of the effects described there have parallels with the topics discussed in this article. The differences in the data are mainly determined by the substantial differences in the ambient pressure in the laser interaction zone. Thus, for example, the size of the actively radiating region for a pressure of $100 \mathrm{~Pa}$ is $\sim 5$ times larger than for standard pressure with other conditions the same. Thus, there are differences in the propagation of the shock wave and in its interaction with the trailing edge of the driver pulse. The continuum emission for standard pressure is localized within distances of 1-2 $\mathrm{mm}$ from the target, while it can be detected at distances up to $11 \mathrm{~mm}$ from the target at reduced pressures. Here (as opposed to [31]) we had the possibility of simultaneously measuring the temporal and spectral dependences of the continuum emission (Fig. 5a). The variations in the intensities of individual analytic spectrum lines (which emit during recombination of the corresponding ions) differ somewhat in [31] on the spatial and time scales, but it is difficult to make a more definitive statement since there are no comparable data for any of the metals.

An analysis of these data may give an impression of an apparent contradiction: why can a typical line spectrum of the sort corresponding to a fairly rarefied plasma [25] be seen in the spectra of Fig. 4 rather than a strong smooth component accompanied by bursts of individual lines? Here this is explained by the way the ELF spectrum is recorded: the instrument is adjusted so that the spectrum is taken from the near-surface region of the target at a height of $3-5 \mathrm{~mm}$ above its surface. At the same time, the continuum from the plasma front is confined to $1-2 \mathrm{~mm}$ from the surface $[9,10]$ and only the further adiabatic expansion of the plasma leads to cooling and rarefaction of this plasma and creates the conditions for production of the line spectra. A slight change in the measurement setup (e.g., in [23]) eliminates this contradiction, but makes it much harder to interpret the spectra. 
An interpretation [22] of the temporal structure of the characteristic lines of $\mathrm{Cu}$ in an ELF has been used to obtain an estimate for the electron temperature similar to that shown in Fig. 5c, curve 3, but with a maximum $500 \mathrm{~ns}$ after the laser pulse. However, the details of the experimental setup did not allow an examination of time intervals less than $200 \mathrm{~ns}$ after the laser pulse. Thus, electron temperature has been estimated there [22] for a plasma actually has been cooled.

The data of [23] are indirect confirmation of the conclusions of this paper. In that case, when the intensity of the driver pulse was increased by an order of magnitude (to $30 \mathrm{GW} / \mathrm{cm}^{2}$ ) the estimated electron temperature was only $50-70 \%$ higher, which is entirely obvious since the influence of the laser parameters on the characteristics of a significantly cooled plasma will be quite weak. Given these remarks, the results in Table 2 must be treated as lower bound estimates. Thus, the actual parameters of an erosion plasma over $1 \mu$ s following the laser pulse are significantly higher than those which can be determined by spectral analysis of individual lines.

Conclusions. The experiments reported here have been used to establish the spectral and temporal form of the emission from erosion laser flares of $\mathrm{Pb}, \mathrm{Zn}$, and $\mathrm{Ni}$, and the corresponding continuum which shows up during initial formation of the flare. For example, in the case of $\mathrm{Pb}$ the initial formation of laser erosion flares during the first 200-300 ns after application of the laser pulse is characterized by continuum emission with a broad peak in the $400-500 \mathrm{~nm}$ range localized at a distance of $1-2 \mathrm{~mm}$ from the target surface.

The experimental data have been used to examine the characteristic lines produced by recombination of ions of the atmospheric gases and the target material. Since these are the data used in plasma diagnostics, it is necessary to distinguish the different stages in the evolution of erosion laser flares used for measuring electron density and temperature. Thus, the appearance of a distinct line spectrum is indicative of the presence of a substantially cooled plasma with a rather low density and temperature. We have shown that the observed continuum emission is superimposed on line emission from near the target surface.

\section{REFERENCES}

1. N. N. Rykalin, A. A. Uglov, and A. N. Kokora, Laser Processing of Materials [in Russian], Mashinostroenie, Moscow (1975).

2. S. I. Anisimov, Ya. A. Imas, G. S. Romanov, and Yu. V. Khodyko, The Effect of High-Power Laser Radiation on Metals [in Russian], Nauka, Moscow (1970).

3. Yu. P. Raizer, The Effect of Laser Radiation [in Russian], Mir, Moscow (1974).

4. L. I. Mirkin, Physical Basis of Materials Processing with Laser Beams [in Russian], Izd. Mosk. Univ., Moscow (1975).

5. A. M. Prokhorov, V. I. Konov, I. Ursu, and I. N. Miheilescu, The Interaction of Laser Radiation with Metals [in Russian], Nauka, Moscow (1988).

6. J. Singh and S. Thakur, Laser-Induced Breakdown Spectroscopy, Elsevier, Amsterdam (2007).

7. N. Kawahara, J. L. Beduneau, T. Nakayama, E. Tomita, and Y. Ikeda, Appl. Phys. B, 86, 605-614 (2007).

8. W. Demtröder, Laser Spectroscopy, Vol. 2, Experimental Techniques, Springer, Berlin, Heidelberg (2008).

9. V. K. Goncharov, K. V. Kozadaev, M. V. Puzyrev, and V. M. Stetsik, J. Eng. Phys. Thermophys., 82, 630-634 (2009).

10. V. K. Goncharov, K. V. Kozadaev, D. V. Shchegrikovich, J. Eng. Phys. Thermophys., 84, 781-786 (2011).

11. M. D. Aksenenko and M. L. Baranochnikov, Optical Radiation Detectors [in Russian], Radio i Svyaz', Moscow (1987).

12. I. K. Kikoin, Tables of Physical Quantities [in Russian], Atomizdat, Moscow (1976).

13. V. K. Goncharov, K. V. Kozadaev, and M. V. Puzyrev, in: M. Sosa and J. Franco (Eds.), Engineering Physics and Mechanics: Analyses, Prediction and Applications, ISBN: 978-1-60876-227-9 (2010), pp. 441-471

14. S. I. Anisimov and B. S. Luk'yanchuk, Usp. Fiz. Nauk, 172, No. 3, 301-333 (2002).

15. T. V. Kononenko, V. I. Konov, S. V. Garnov, R. Danelius, A. Piskarkas, G. Tamosauskas, and F. Dausinger, Kvant. Élektron., 28, No. 2, 167-172 (1999).

16. E. Yu. Loktionov, A. V. Ovchinnikov, Yu. Yu. Protasov, and D. S. Sitnikov, Zh. Prikl. Spektrosk., 77, No. 4, 604-611 (2010).

17. V. K. Goncharov and K. V. Kozadaev, J. Eng. Phys. Thermophys., 83, No. 1, 90-95 (2010).

18. K. V. Kozadaev, Perspektivnye Materialy, 6, 71-78 (2011).

19. F. V. Bunkin, A. E. Kazakov, and M. V. Fedorov, Usp. Fiz. Nauk, 107, No. 4, 559-593 (1972).

20. A. K. Shuaibov, M. P. Chuchman, and L. L. Shimon, Tech. Phys. Lett., 30, No. 24, 49-55 (2004).

21. M. P. Chuchman and A. K. Shuaibov, Plasma Phys. Rep., 34, No. 4, 340-346 (2008). 
22. V. K. Unnikrishnan, A. Kamlesh, V. B. Kartha, C. Santhosh, G. P. Gupta, and B. M. Suri, Pramana - J. Phys., 74, No. 6, 983-993 (2010).

23. M. Kubkowska, P. Gasior, M. Rosinski, J. Wolowski, M. J. Sadowski, K. Malinowski, and E. Skladnik-Sadowska, Eur. Phys. D, 54, 463-466 (2009).

24. V. S. Burakov, A. V. Butsen', and N. V. Tarasenko, Zh. Prikl. Spektrosk., 77, No. 3, 416-424 (2010).

25. H. Griem, Plasma Spectroscopy [Russian translation], Atomizdat, Moscow (1969).

26. V. N. Kolesnikov, Spectroscopic Diagnostics of Plasmas [in Russian], MIFI, Moscow (2007).

27. N. Kumar, S. Dash, A. K. Tyagi, and R. Baldev, Sadhana, 35, No. 4, 493-511 (2010).

28. A. N. Zaidel', V. K. Prokof'ev, and S. M. Raiskii, Tables of Spectrum Lines [in Russian], Izd. Tekh.-Teoret. Lit, Moscow (1952).

29. C. Corliss and W. Bozeman, Transition Probabilities and Oscillator Strengths for 70 Elements [Russian translation], Mir, Moscow (1968).

30. J. E. Sansonetti and W. C. Martin, J. Phys. Chem. Ref. Data, 34, No. 4, 1559-2259 (2005).

31. T. Sukhov, Laser Spectral Analysis [in Russian], Nauka, Novosibirsk (1990). 